\title{
Heart-pulse Biofeedback in Playful Exercise using a Wearable device and Modular Interactive Tiles
}

\author{
Tomoya Shimokakimoto* \\ Graduate School of Systems \& Information Engineering, University of Tsukuba, \\ 1-1-1 Tennodai, Tsukuba 305-8573, Japan \\ Henrik Hautop Lund \\ Centre for Playware, Technical University of Denmark, \\ 2800 Kgs. Lyngby, Denmark \\ Kenji Suzuki \\ Center for Cybernics Research, University of Tsukuba, \\ 1-1-1 Tennodai, Tsukuba 305-8573, Japan \\ / Japan Science and Technology Agency, \\ Kawaguchi Center Building 4-1-8, Honcho, Kawaguchi 332-0012, Japan \\ *Corresponding author. E-mail: shimokakimoto@ai.iit.tsukuba.ac.jp \\ Tel: $+81-29-853-5679$
}

\begin{abstract}
We developed a playful biofeedback system using a wearable device and modular interactive tiles. In this approach we suppose that patients could regulate exercise intensity on their own through biofeedback. We propose biofeedback play system called "bioToys" based on exercise with the modular interactive tiles. The system consists of a wearable device that measures heart-pulse via ear-mounted sensor, and modular interactive tiles which are used for physical rehabilitation exercise through playing a game. The wearable devise enables detection of heart pulse in real-time and therefore provides heart beat rate during playful activities, even if the heart pulse wave have motion artifacts. The tiles are designed to build flexible structures and to provide immediate feedback based on the users' physical interaction with the tiles. We combine the two systems to provide users with heart pulse biofeedback in playful exercise. We show that using the developed system it is possible for the users to regulate the exercise intensity on their own with biofeedback, and also possible to analyze exercise activity using number of steps on the tiles and heart beat rate.
\end{abstract}

Keywords: playware, biofeedback, exercise, rehabilitation 


\section{Introduction}

In order to improve the health and quality of life (QOL) of elderly people and patients with metabolic disorders, stroke and cardiac diseases, physical training and exercise is required in daily life. Heart Beat Rate (HBR) is usually used as a standard for regulating the exercise intensity ${ }^{1}$. Thus, there are some commercial wearable devices of measuring HBR. And some exercise systems that control the exercise intensity based HBR are worth consideration ${ }^{2,3}$.

For practical implementation of wearable sensing technologies in physical exercise, there is a n eed for methods to simplify measurement of bio-signals, as well as signal processing techniques to reduce motion artifacts in order to provide stable biofeedback. Systems such as those in Refs.4 - 5 try to take those considerations into account.

In physical exercise, it is not easy to maintain participants' motivation for joining a sport. ${ }^{6}$ Recent researches also utilized games as means for exercise, showing a number of cases where gaming may be beneficial for health.

In this paper, we propose the hypothesis that patients could regulate exercise intensity on their own through biofeedback during playful activity, and we propose a biofeedback play system called "bioToys" based on exercise with a wearable device and the modular interactive tiles.

\section{System Configuration}

\subsection{Wearable Device}

We developed a wearable device to measure heart pulse and generate biofeedback in real-time. The wearable device consists of an ear-mounted sensor (ear sensor, Combi Wellness Corp.), a processor (LPC1752FBD80, NXP), a light emitting diode for biofeedback and Bluetooth module for communication with PC. Fig. 1 shows the overview of the wearable device for heart pulse measurement and biofeedback.

Heart pulse measurement via ear-mounted sensor is prone to noise resulting from body movements, thus it is important to process the heart pulse waveform in realtime to generate a s table biofeedback output. In our device we implemented an algorithm based on linear prediction and an adaptive template matching method to

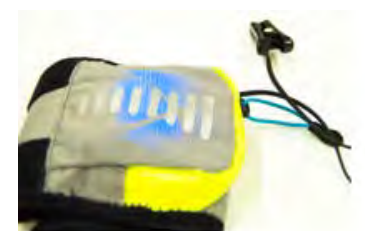

(a)

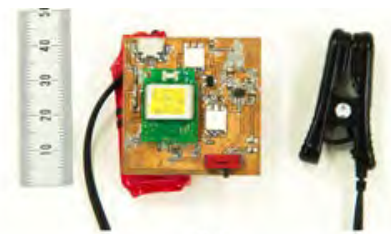

(b)
Fig.1. (a) Overview of the wearable device in a sports wrist pocket (b)the circuit board and heart pulse sensor

overcome noise in the waveform ${ }^{5}$. The algorithm is capable of detecting actual edges in the heart pulse waveform in real-time, even if the pulse waveform include motion artifacts similar to heart pulse. This method utilizes human physiological features to generate a correct cyclic pulse from noisy signals. This algorithm is mainly constructed by two equations (see equation 1 a nd 2). The equation 1 represents a differential equation of linear prediction and the equation 2 means a template matching.

In equation $2, P(t)$ is the pulse waveform vector measured by the $\mathrm{A} / \mathrm{D}$ converter with the mean value normalized to zero. When $P$ includes a cyclic motion artifact such as those resulting from locomotion or jumping, a mere thresholding method is not adequate to distinguish such artifacts from heart pulse. The developed algorithm, by linear prediction, avoids detecting artifacts similar to heart pulse. In the linear prediction method we designed a Kalman filter according to equation 1 , which is a differential equation model for calculating an estimate time interval of the heart pulse $\quad[\mathrm{s}]$ depending on previous detected time intervals. This linear prediction method is capable of approximately predicting the next heart pulse interval according to the pattern of the heart pulse ${ }^{7}$. Therefore, an edge of heart pulse waveform is detected after $\hat{\tau}_{c}(m)+\delta_{t}[\mathrm{~s}]$, which is the sum of estimated time and difference between the previous estimated time and actual detected pulse interval.

In addition, for reducing noise effect around an edge of heart pulse waveform, this algorithm uses ideal pulse waveform vectors for heart rates $\left(\tau_{s}[\mathrm{bpm}]\right)$ that don't include noise: $I\left(t ; \tau_{s}\right)$. Then, using the correlation value between $I$ and $P ; M\left(\tau_{s}\right)$ in equation 2, when the value of $\mathrm{M}(\tau)$ is higher than a threshold, the system detects an edge of heart pulse waveform. 
Using the linear prediction and template matching methods, this wearable device is able to detect edges of heart pulse and calculate HBR stably. LEDs on the device blink in synchronization with the detected heart pulse, and the HBR data is transmitted to PC via Bluetooth in real-time.

$$
M\left(\tau_{s}\right)=\frac{1}{N} \sum_{i=0}^{N-1} I(t-n \tau) P(t-n \tau)
$$

\subsection{Modular Interactive Tiles}

We developed a system for playful biofeedback based on modular interactive tiles. The tiles attach to each other to form the overall system, and they are designed to be flexible for different arrangements. The tiles are also motivating to the user since they provide immediate feedback based on the users' physical interaction with the tiles, which is a design principle of modular playware ${ }^{8}$. F ig. 2 shows a scene of playing with the tiles.

Each tile comprises a sensor and transmitter for communicating with other tiles, has a quadratic shape measuring $300 \mathrm{~mm} * 300 \mathrm{~mm} * 33 \mathrm{~mm}$, a force sensitive resistor (FSR) and eight RGB LEDs that emit light with equal spacing in between on a ci rcle shape for game feedback

On the circuit board of each tile there are also connectors to mount an XBee radio communication add-on board, including the MaxStream XBee radio communication chip. Hence, there are two types of tiles, those with a radio communication chip (master tiles) and those without one (slave tiles). The master tile may communicate with the game selector box and initiate the games on the built platform. Every platform has to have at least one master tile if communication is needed to game selector box or a PC. The tile becomes a master tile only when it has XBee radio communication connected to it.

The modular interactive tiles can be easily set up on floor. They can be simply attached to each other as a jigsaw puzzle, and there are no wires. The modular

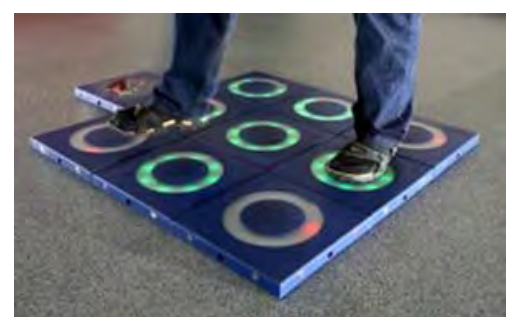

Fig.2. Modular interactive tiles used for playful physiotherapy with feet

interactive tiles can register whether they are placed horizontally or vertically, and they can adapt the software games to behave accordingly.

\subsection{Real-time biofeedback for exercise system}

The games on modular tiles are designed for physical exercise. B asically when users touch or step on the tiles, the tiles generate feedback through LEDs' color and intensity according to the game's rules that are installed in tiles. We developed a playful biofeedback system by combining a wearable device for measurement of heart pulse rate with the modular interactive tiles. In this paper, we modified the games installed in modular interactive tiles ${ }^{9}$ for some new purposes as will be illustrated.

The wearable device transmits the calculated HBR to $\mathrm{PC}$ via Bluetooth as the wearable device detects users' heart pulse. After the PC receives data of HBR, data is logged in the PC and transmitted to the master tile wirelessly via XBee. When the master tile receives the heart beat data, it in terprets it and light the LEDs according to the game rules. This system has six colors for biofeedback. Fig. 3 shows an example of color assignment based on HBR in reference to ratings of perceived exertion scale, which is a scale of perceived physical exertion relating to $\mathrm{HBR}^{10}$.

For biofeedback, HBR is assigned by color and the game level or score point. Modular interactive tiles have games based on counting steps or touches, or based on time constraints. For example, there are "color race game" and "final countdown". Color race is based on counting steps. When the user steps on an activated or illuminated tile, user gets score. Finally when the user acquires an adequate score the game finishes successfully. By adding the wearable heart beat device, the user is able to know his heart pulse and HBR and share it with audience. 


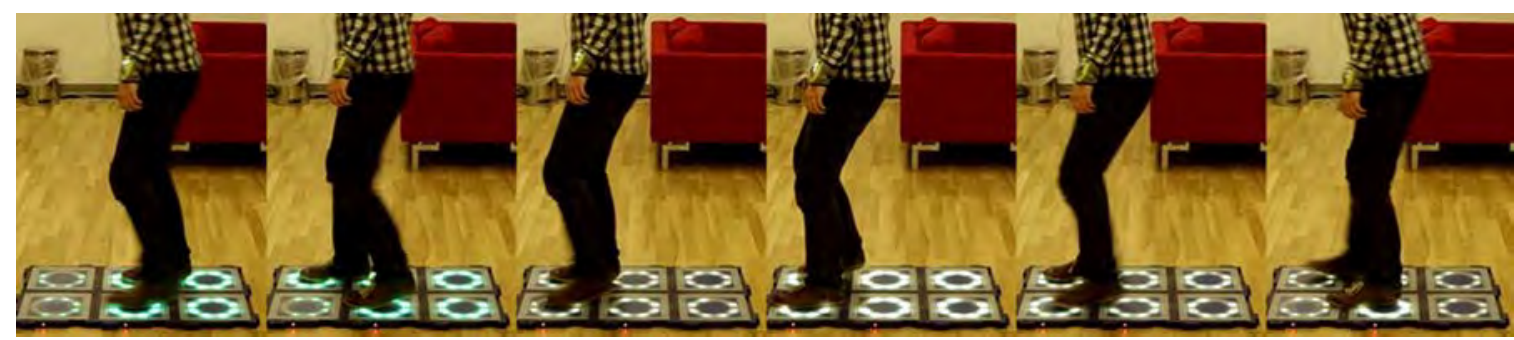

Fig.4. The scene in the playing using a wearable device and modular interactive tiles: Tiles' LED color was changed from green to yellow depending on user's HBR value.

Final countdown game is based on time constraint. In this game, all tiles are activated and the eight LEDs on each tile count down independently. When any tile finishes to count down it will send a "game over" message to the master tile and the game will finish. When the user steps on a tile, count down time in this tile will reset and the eight LEDs on this tile are lit again. For this game, in normal case, count down speed parameter is fixed depending on game difficulty. For regulating the exercise, we designed a game where the user keeps playing as long as proper HBR is observed. In this case, we designed the countdown parameter to change depending on the HBR. At the first level that is under $70 \mathrm{bpm}$ (color is purple), the counts down interval is $2 \mathrm{~s}$. Then for the following levels (light blue, blue, green) the countdown time decreases 0.32 s respectively. Considering the fourth level (green, 100bpm) as the desired level, count down timer will increase for later levels (yellow and red) also by 0.32 s respectively, in order to keep the user at the desired level.

\section{Experiment}

\subsection{Regulating exercise}

In order to evaluate the performance of the proposed system, we conducted an evaluation experiment to verify the feasibility of the proposed system as a playful biofeedback system for regulating exercise. In this experiment, we used six tiles and final countdown game. We asked four healthy male participants to exercise for two minutes with the

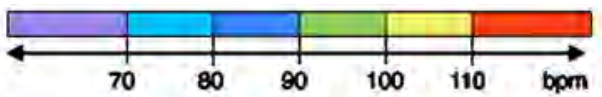

Fig.3. Example of a color assignment based on HBR proposed system and to go up to the third level (green color level) and keep this level. We logged heart beat pulse by $120 \mathrm{~Hz}, \mathrm{HBR}$, the number of steps and also recorded these experiments on video for analysis.

\subsection{Result and analysis method}

Fig. 4 shows a scene during the experiment. We confirmed that the proposed system provide color feedback dependent on HBR during the game. Fig. 5 shows all participants' results of this experiment. Here we compare the number of steps and HBR among participants. In this experiment, the results of participant $\mathrm{A}$ and $\mathrm{B}$ were similar. Participant $\mathrm{C}$ (who exercises on regular bases) did not get tired during the experiment. On the other hand, participant D who does not usually exercise was tired from the point of HBR.

Then we focused on the time series of steps and HBR. Fig. 6 and 7 show the results of two participants (A and B). The top graph shows the events of detected heart pulse, the HBR is expressed in the color of biofeedback. The bottom graph shows the sum value of number of steps for each 10 seconds.

In Fig.6, when participant $\mathrm{A}$ reached green

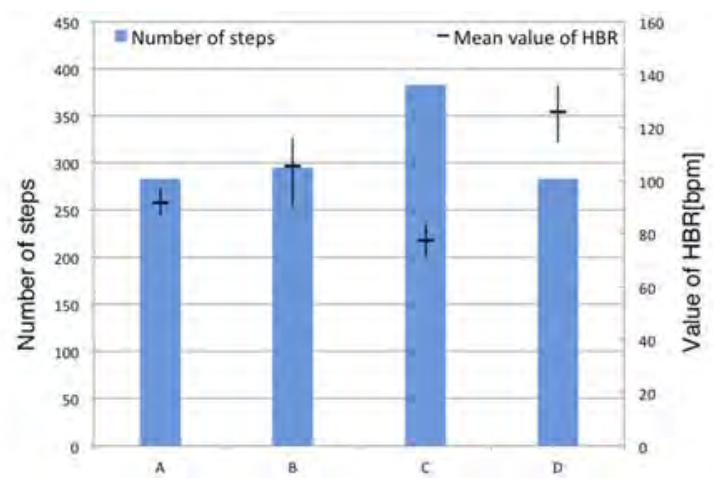

Fig.5. The result of all participants 
level, he intentionally decreased his pace to the desired HBR. In participant B's case, first he overshoot to the red level for around 20s, then he decreased his pace to keep the desired HBR. Finally, he was able to find the proper pace to stay between green and yellow levels. The proposed system showed the possibility for the users to regulate the exercise intensity during playful activity on their own with biofeedback.

\section{Conclusion}

In this paper we proposed a biofeedback play system called "bioToys" based on exercise with modular interactive tiles and a wearable device. We consider that games as a mean for exercise are beneficial for health, and for maintaining the motivation for exercise.

We developed a wearable device that measures heart pulse via ear-mounted sensor, and we implemented an algorithm for biofeedback in the real time which detects heart pulse in intense activity. We also developed a system for playful feedback game based on modular interactive tiles. Then we combined the two systems and developed games to provide users with heart pulse biofeedback in playful exercise.

We devised an evaluation experiment to verify the feasibility of the proposed system for regulating exercise. We showed that it is possible to use the

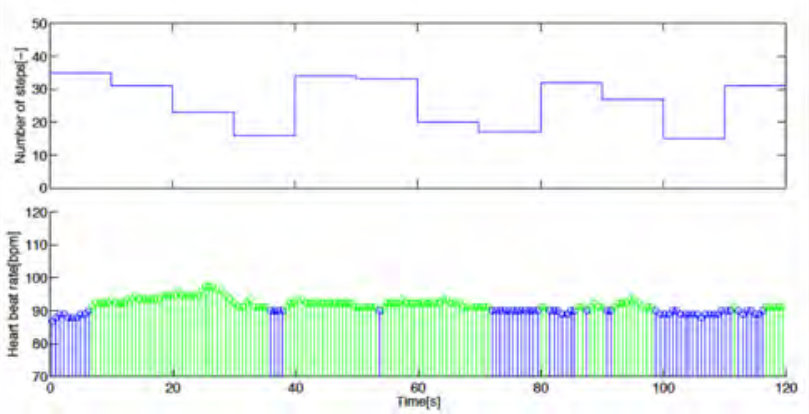

Fig.6. The result of participant $\mathrm{A}$ in two minutes exercise

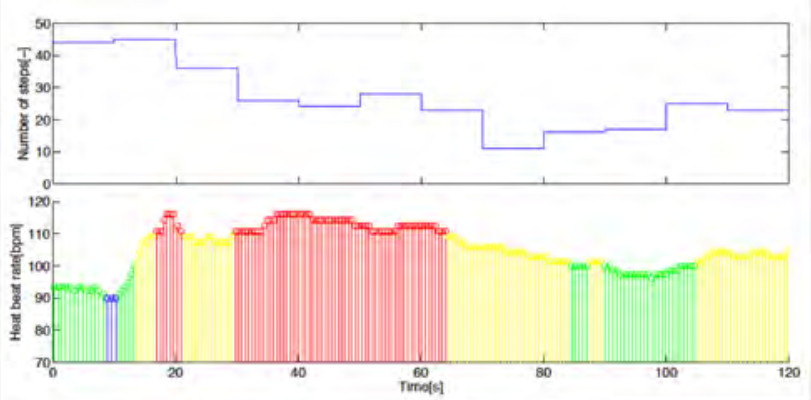

Fig.7. The result of participant B in two minutes exercise developed system to regulate the exercise intensity on one's own with biofeedback, and it is also possible to analyze exercise activity using number of steps on the tiles and HBR.

\section{References}

1. Karvonen, J., and Vuorimaa, T., Heart Rate and Exercise Intensity During Sports Activities., Sports Medicine, 5(5) (1988) 303-311.

2. Su, S., W., and Wang, L., and Celler, B., G., and Savkin, A., V., Heart rate control during treadmill exercise. Proc. of Annual Intl. Conf. of the IEEE EMBS (China, Shanghai, 2005), pp. 2471-2474.

3. ElSamahy, E. and Genedy, A. and Abbass, M.A. and Gaddallah, M., A Computer-based System for Safe Physical Fitness Evaluation, Proc. of Annual Intl. Conf. of the IEEE BMEI (USA, Boston 2011), pp. 1443-1447.

4. Watanabe, M., and Tsukamoto, S., and Hoshino, H., A handle electrode system for measuring heart rate while riding a bicycle, Proc. of Annual Intl. Conf. of the IEEE BMEI (Canada, Vancouver, 2008), pp. 542-545.

5. Shimokakimoto, T., and Ayuzawa, S., and Suzuki, K., Real-time Pulse Detection for Physiothrapy and its Application toWearable Device, IPSJ Journal, 54(4) (2013) 1480-1488 (in Japanese).

6. Dishman, R.K., Exercise adherence: Its impact on public health, Human Kinetics Publishers. (1988).

7. Kobayashi, M., and Musha, T., 1/f Fluctuation of Heartbeat Period, IEEE transactions on biomedical engineering, 29(6) (1982) 456-457.

8. Lund., H., H., and Marti, P., Designing modular robotic playware, the IEEE Int. Workshop Robots Human Interactive Commun (Japan, Toyama, 2009), pp. 115121.

9. Lund., H., H., Modular Interactive Tiles for Rehabilitation: Evidence and Effect, Proc. of the 10th WSEAS Intl. Conf. on ACS, (Japan, Iwate, 2010), pp. 520-525.

10. Borg, G., Perceived exertion: a note on "history" and methods., Med. Sci. Sports., 5(2) (1973) 90-93. 